\title{
La formation postgraduée dans les hôpitaux est fortement appréciée
}

\author{
Bernadette Sütterlin ${ }^{a}$, Lea Christina Burgermeister ${ }^{b}$, Michael Siegrist $^{c}$, Werner Bauer $^{d}$ \\ ${ }^{a}$ Dr sc., Senior Researcher; ${ }^{b}$ lic. phil., collaboratrice scientifique; ' Prof. Dr phil., professor for Consumer Behavior à I'ETH Zurich; \\ ${ }^{\text {d }}$ Dr méd., président de I'ISFM
}

\section{Introduction}

Une formation postgraduée de haute qualité requiert l'engagement des formateurs. Or, de plus en plus de voix critiques s'élèvent aujourd'hui pour montrer du doigt le déclin du rôle central de la formation postgraduée dans les hôpitaux ainsi que le manque de soutien et de reconnaissance dont souffrent les médecinscadres un peu partout quant à leur engagement dans la formation postgraduée. Les directions hospitalières, par exemple, seraient de moins en moins disposées à créer les conditions-cadres nécessaires à une formation postgraduée de haute qualité. Il est donc à craindre que la formation postgraduée ne bénéficie plus de l'importance requise avec, à la clé, une baisse inévitable de sa qualité.

Afin de garantir une formation postgraduée efficace et de haut niveau, les responsables d'établissement doivent disposer, hormis l'engagement nécessaire, des compétences requises pour l'exercice des diverses fonctions leur incombant. Ces compétences regroupent non seulement les qualifications spécifiques, mais également des aptitudes didactiques et des compétences en gestion et en management. Une préparation optimale au rôle de responsable d'un établissement de formation postgraduée et à l'exercice de ses fonctions facilite la transmission de la direction d'un établissement et constitue la base d'une formation postgraduée de haute qualité dès le début. Il est donc capital d'identifier d'éventuelles lacunes dans les compétences requises pour l'exercice de la fonction de responsable d'établissement afin de pouvoir y remédier ultérieurement par des offres de formation ciblées.

En vue d'évaluer l'importance de la formation postgraduée et d'identifier d'éventuels déficits dans la préparation à la fonction de responsable d'établissement, des questions spécifiques supplémentaires ont été posées aux responsables d'établissement dans le cadre de l'enquête statistique annuelle. Le recensement des données s'est étendu sur une période allant d'avril à août 2016. Le relevé statistique qui a lieu chaque année préalablement à l'évaluation de la formation postgraduée par les médecins-assistants est réalisé par l'ETH Zurich sur mandat de l'Institut suisse pour la formation médicale postgraduée et continue (ISFM). Dans le cadre de cette enquête, les responsables d'établissement ont la possibilité de s'exprimer sur des thèmes de politique sanitaire actuels. En 2016, cette partie variable du questionnaire portait sur «l'importance de la formation postgraduée» et la "préparation à un rôle dirigeant».

\section{Méthode}

\section{Participants et réalisation}

Dans le cadre du recensement statistique des postes de formation postgraduée au printemps 2016, les responsables d'établissement ont reçu un lien vers un bref questionnaire en ligne. 1440 d'entre eux ont répondu aux questions supplémentaires de la partie variable de ce questionnaire qui font l'objet du présent article ce qui correspond à un taux de réponse de $93 \%$.

\section{Questionnaire}

La première partie de la partie variable du questionnaire portait sur la préparation à un rôle dirigeant. Dans un premier temps, il a été demandé aux responsables d'établissement s'ils se sentaient bien préparés aux différentes tâches au moment de leur entrée en fonction puis, dans un second temps, s'ils s'estimaient compétents aujourd'hui dans leur fonction. Dans la seconde partie de la partie variable sur l'importance de la formation postgraduée, les responsables d'établissement ont été priés d'évaluer l'importance qui est accordée à la formation postgraduée dans leur hôpital. Ensuite, ils ont été interrogés sur leur identification avec divers rôles. Chaque question exigeait une évaluation sur une échelle de 1 à 6 .

\section{Résultats}

\section{Préparation à un rôle dirigeant}

Lors de leur entrée en fonction, les responsables d'éta- 
blissement de formation postgraduée se sont sentis le mieux préparés - parmi les différentes tâches de direction - à la fonction de responsable/décideur médical.

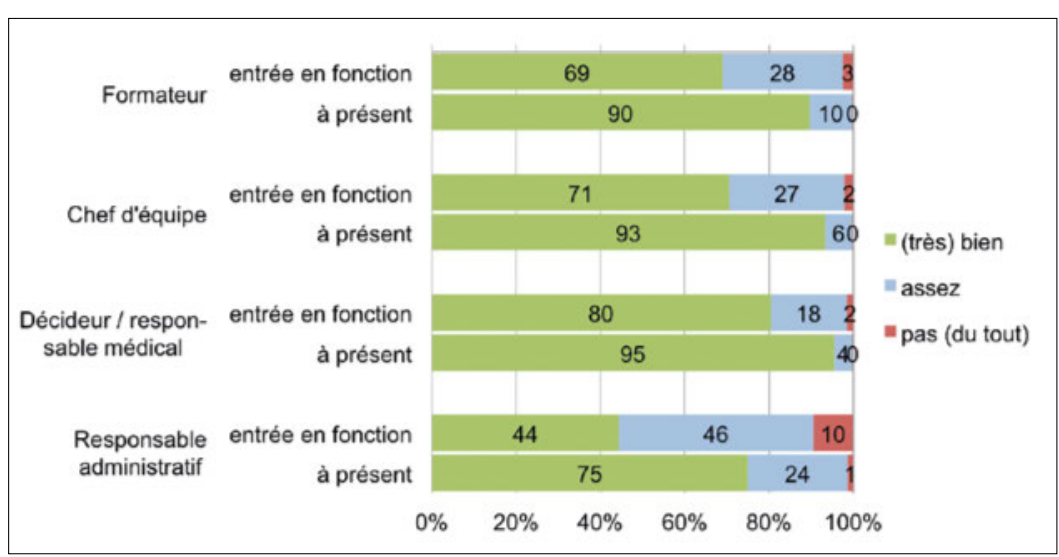

Figure 1: Indications fournies par les responsables d'établissement de formation postgraduée concernant la perception de leur préparation/compétence dans les différentes fonctions d'un responsable d'établissement lors de leur entrée en fonction et à présent ( $\mathrm{N}$ variait de 1430 à 1436).

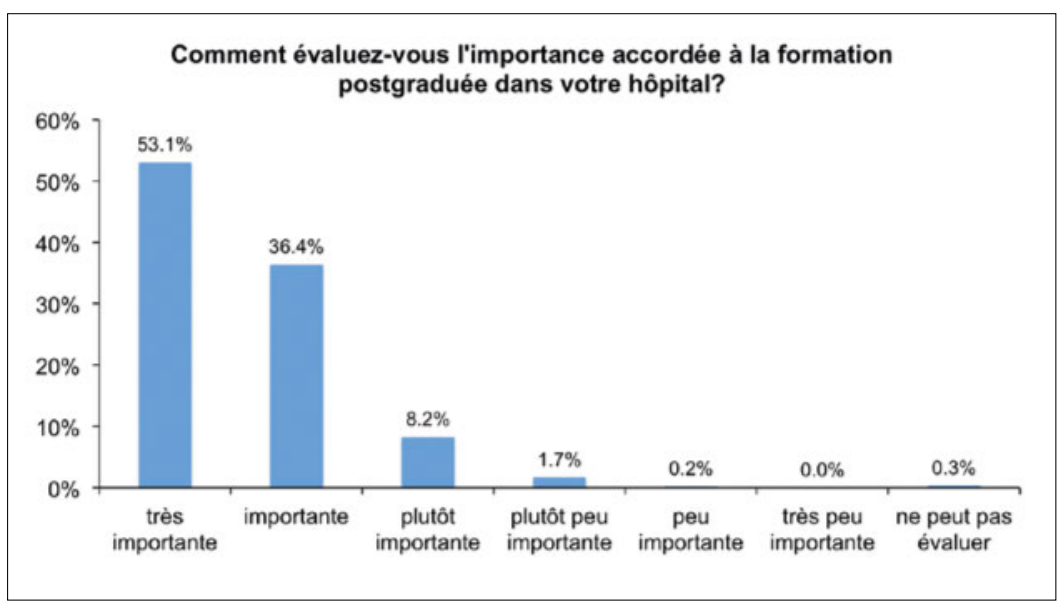

Figure 2: Evaluation par les responsables d'établissement de formation postgraduée de l'importance accordée à la formation postgraduée. La fréquence des réponses est indiquée en pourcentage $(\mathrm{N}=1433)$.

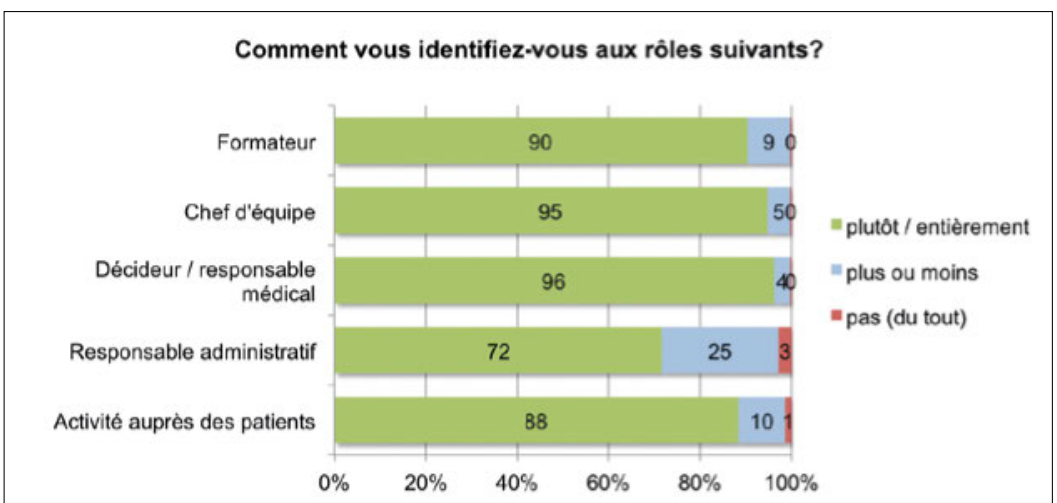

Figure 3: Indications fournies par les responsables d'établissement de formation postgraduée sur le degré d'identification aux différents rôles d'un responsable d'établissement ( $N$ variait de 1430 à 1434)
Pour chacun des rôles de formateur et de chef d'équipe, un petit tiers des personnes interrogées s'est senti «moyennement» ou «mal préparé». Avec l'expérience professionnelle, leurs propres compétences liées à ces fonctions sont mieux perçues et une grande majorité des responsables d'établissement les juge à présent (très) bonnes. En revanche, rares sont les responsables d'établissement qui se sentaient bien préparés au rôle de responsable administratif lors de leur entrée en fonction. Une petite moitié se considérait «assez» compétente et $10 \%$ des personnes interrogées «pas du tout». Même si les aptitudes à la fonction de responsable administratif s'améliorent avec l'expérience, un quart environ des responsables d'établissement s'estime encore «assez» ou "pas (du tout)» compétent pour cette fonction. Les résultats concernant la préparation au rôle de responsable d'établissement de formation postgraduée sont représentés dans la figure 1.

\section{Importance de la formation postgraduée}

Contrairement à certaines suppositions, la place accordée à la formation postgraduée dans leur propre hôpital est jugée "très importante" par la moitié des responsables d'établissement (fig. 2). Les autres $40 \%$ la jugent «importante» ou "plutôt importante». Rares sont les responsables d'établissement estimant que la place accordée à la formation postgraduée dans leur hôpital est «peu importante» ou «plutôt peu importante» $(<2 \%)$.

L'identification aux différents rôles d'un responsable d'établissement de formation postgraduée est en général très élevée (fig. 3). La majorité des responsables d'établissement s'identifient fortement à leur rôle de responsable/décideur médical, à leur fonction d'activité auprès des patients ainsi qu'à leurs rôles de chef d'équipe et de formateur. En revanche, ils s'identifient beaucoup moins au rôle de responsable administratif.

\section{Discussion}

Un tiers des responsables d'établissement de formation postgraduée interrogés se sentaient mal préparés à leur rôle de formateur et de chef d'équipe lors de leur entrée en fonction. En ce qui concerne le rôle de responsable administratif, ce chiffre s'élève même à plus de la moitié des personnes interrogées. Un quart des responsables d'établissement ne s'identifie toujours pas, avec le temps, au rôle de responsable administratif. Ces résultats révèlent qu'il serait primordial d'améliorer la transmission de "soft skills» comme les aptitudes didactiques et de communication, la conduite de personnel, l'économie et la gestion. Le niveau des compétences médicales semble en revanche permettre une 
parfaite transmission de la fonction de responsable/ décideur médical d'un établissement de formation postgraduée.

Les présents résultats concernant l'acquisition de compétences par les responsables d'établissement correspondent à ceux d'une enquête menée en 2014 auprès de médecins spécialistes pour évaluer la formation postgraduée. Ces derniers avaient estimé qu'au moment de leur entrée en fonction, ils présentaient également un déficit dans l'acquisition de "soft skills" durant la formation postgraduée en vue du titre de spécialiste [1]. Même si la Réglementation pour la formation postgraduée de l'Institut suisse pour la formation médicale postgraduée et continue (ISFM) comporte une série d'objectifs de formation généraux (par ex. communication, conduite de personnel, éthique, principes économiques, gestion des erreurs), leur transmission n'est pas simple à réaliser dans l'actuel quotidien hospitalier et des moyens doivent être recherchés pour l'amé-

liorer.

Institute for Environmental

Decisions (IED)

Consumer Behavior

ETH Zürich CHN I 76.3

Universitätstrasse 22

CH-8092 Zurich
Contrairement à la faible importance redoutée de la formation postgraduée dans les établissements de formation postgraduée, les responsables d'établissement s'identifient fortement à leur rôle de formateur et considèrent qu'une place importante est accordée à la formation postgraduée dans leur hôpital. Ainsi, les résultats de l'enquête ne confirment pas les inquiétudes exprimées sur le remplacement des médecins en formation par des médecins hospitaliers. Les établissements de formation postgraduée continuent de s'identifier à leur mandat de formation et cette identification semble l'emporter sur les considérations de coûts liés au recrutement de médecins hospitaliers. Même dans le cadre de l'introduction du système DRG et de la pression économique qui y est liée, pratiquement aucun cas n’a été enregistré dans lequel des médecins en formation auraient été remplacés par des médecins hospitaliers - contrairement à ce qu'avaient annoncé de nombreux responsables d'établissement [2].

\section{Crédits illustration}

Mises à disposition par les auteurs

\section{Références}

1 Siegrist M, Sütterlin B, Burgermeister LC, Bauer W. Evaluation de la formation postgraduée par les médecins spécialistes. Bulletin des médecins suisses. 2015;96(22):758-63.

2 Sütterlin B, Burgermeister LC, Siegrist M, Bauer W. Résultats de l'enquête 2014: Evaluation de la formation postgraduée. Bulletin des médecins suisses. 2016;97(5):168-71. 